\title{
Analysis on Indian Education Towards Fully Automated Digitization and Decentralized Education System- A Machine Learning Approach
}

\author{
Dillip Narayan Sahu1, Pankajini Sahu ${ }^{2 *}$ \\ ${ }^{1}$ Lecturer, Department of MCA, Gangadhar Meher University (GMU), India \\ ${ }^{2}$ Lecturer, Department of Education, ShreeRam College, Rampur, Sonepur, Odisha, India
}

\begin{abstract}
Education is the major aspect and basis of foundation development in any country. Education directs the right path as well as to build a good human character with all aspect of life. In 2018, India ranked $40^{\text {th }}$ with the score 41.2 . In the year 2019, India was on $35^{\text {th }}$ rank on the overall index with a score of 53. This score was based on policy environment, teaching environment and socio-economic environment. National Education Policy NEP-2021 is mainly focused on skill based education system with more flexible learning for the students. Digitization is a way for speed up of the learning process of the students. In this paper, we have analyze the Indian education system in the context of auto digitization and decentralized control through different machine learning data analysis, prediction, feature extraction, classification, visualization tools.
\end{abstract}

Keywords: Algorithm, Decentralized Education, Digitization, Machine Learning.

\section{INTRODUCTION}

"Any activity wherever we are able to modify and decompose the worth chain, and move the work around, can get stirred around. Some individuals can say, 'Yes, however you cannot serve Maine a cut of meat.' True, however I will take the reservation for your table sitting anyplace within the world,"

Digital education is not about the videos or lectures on whiteboards by lecturers on the net. It's regarding applicable infrastructure, platforms, technology, tools, interactivity, content etc. we have not yet completely prepared for fully atomization in education. Government universities and colleges don't have the sufficient resource to supply digital education. Private educational sectors are totally different. In India, it will be a very complex task to implement fully digitized educational environment and infrastructures for all educational sectors[1][2].

Globalizations impact on institutional reform methods i.e. competitiveness-driven reforms. The reforms is classified into four categories - Decentralization, Standards, Improved management of institutional resources and Improved teacher enlisting and coaching.

Globalizations impact on academic reform methods i.e. competitiveness-driven reforms Decentralization- The purpose is to extend the management over curriculum and teaching ways of native communities and therefore the academics and principals of the schools themselves. An extension of such reforms is faculty selection and the privatization of academic delivery Standards[3].

The main focus of centralization reforms are that the quest for higher learning standards. Learning customary that an academic programme aims to assist learner's attention. The purpose of providing such standards (established by a central authority) is to administer clear signals of educational expectations to varsities and to folks within the hope, high standards can raise parent demands and school performance, Improved management of academic resources. Introducing new, high-yield resources that may make associate degree particularly giant distinction in student achievement at comparatively low value[4][5]. Better management and allocation of existing resources in colleges. Public education in developing countries ought to focus on increasing and rising basic education because the pay-off -the social rate of return to resources invested with at that level is higher than to resources invested with at the secondary and higher levels. 


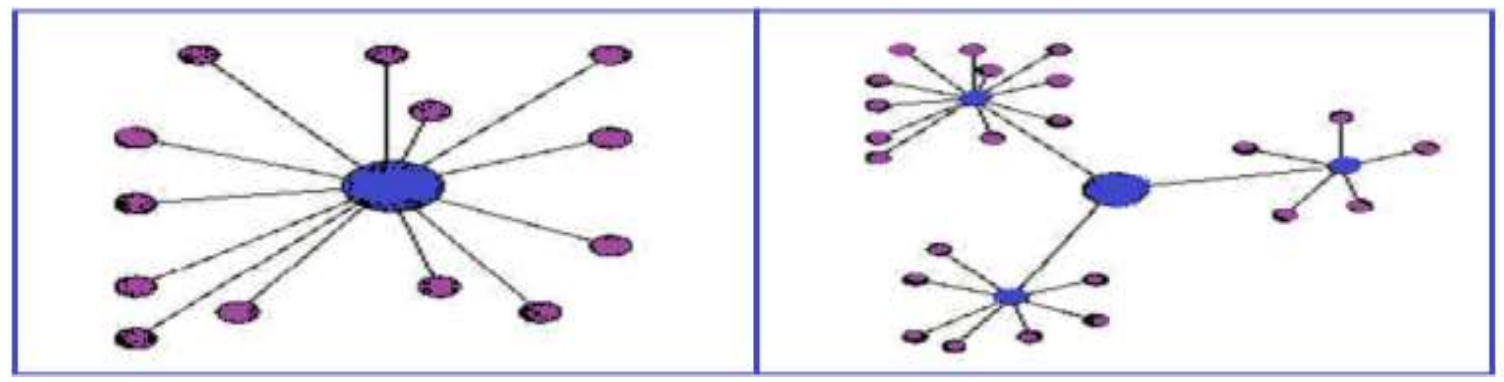

Fig.1 Centralized and Decentralized Education System

Government Universities and Colleges may opt for a completely decentralized implementation process which may not be completely implementable practically but may be possible gradually. Creation of syllabus, course content, practical content, based on current industry standard and market standard which are globally acceptable should be adaptable[6]. So Decentralization means the approach for each school which control by the Government partially only in the aspect of managerial task[7]. The country not only benefits from a wide diversity of theoretical and practical implementation strategies, but it is also able to run these processes simultaneously. Professors, faculties or any academician are afforded maximum opportunity for creativity in their research input output and thus may also have the way to take a new movement towards a new technology for the future[8]. Students benefit from different new technologies and build their mindset for the futuristic development and also have opportunity to be part of the future development process[9].

\section{EXPERIMENTS AND OBSERVATIONS}

We have taken a dataset name=percentage-of-schools-with-comps-2013-2016.csv.arff (Attribute Relation File Format) as our input and generate different results based on different machine learning algorithms.

\subsection{Classifier NAME=weka.classifiers.functions.MultilayerPerceptron}

SYNOPSIS=A classifier that uses backpropagation to learn a multi-layer perceptron to classify instances. The network can be built by hand or or set up using a simple heuristic. The network parameters can also be monitored and modified during training time. The nodes in this network are all sigmoid (except for when the class is numeric, in which case the output nodes become unthresholded linear units).

Classifier Output $===$ Run information $===$

Scheme: $\quad$ weka.classifiers.functions.MultilayerPerceptron -L 0.3 -M 0.2 -N 500 -V 0 -S 0 -E 20 -H a

Relation: percentage-of-schools-with-comps-2013-2016-weka.filters.AllFilter-weka.filters.MultiFilter-

Fweka.filters.AllFilter

Instances: 110 Attributes: 13

State_UT year Primary_Only

Primary_with_U_Primary_Sec_HrSec

U_Primary_With_Sec_HrSec

U_Primary_With_Sec

Sec_with_HrSec.

All Schools

Test mode: 10-fold cross-validation

$===$ Cross-validation $======$ Summary $===$

Correlation coefficient $\quad 0.9944$

Mean absolute error $\quad 2.0938$

Root mean squared error $\quad 2.9674$

Relative absolute error $\quad 9.222 \%$

Root relative squared error $\quad 10.7302 \%$

Total Number of Instances $\quad 110$

\author{
Primary_with_U_Primary \\ U_Primary_Only \\ Primary_with_U_Primary_Sec \\ HrSec_Only
}




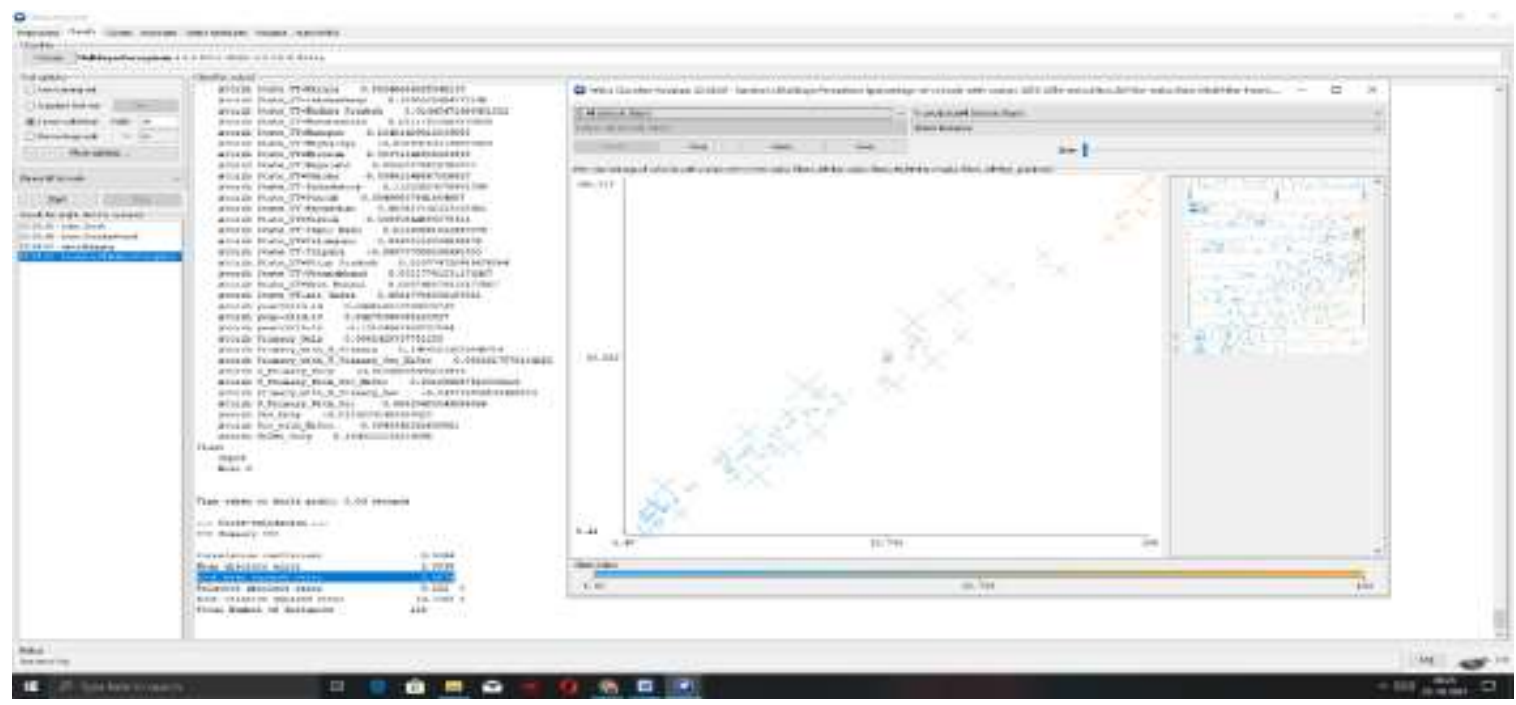

Fig.2 MultilayerPerceptron Classifier with Output Visualization

\subsection{Classifier NAME=weka.classifiers.meta.Bagging}

SYNOPSIS=Class for bagging a classifier to reduce variance. Can do classification and regression depending on the base learner.

Classifier Output $===$ Run information $===$

Scheme: weka.classifiers.meta.Bagging -P 100 -S 1 -num-slots 1 -I 10 -W weka.classifiers.trees.REPTree -- -M 2 -V 0.001 -N 3 -S 1 -L - 1 -I 0.0

Relation:

percentage-of-schools-with-comps-2013-2016-weka.filters.AllFilter-weka.filters.MultiFilter-

Fweka.filters.AllFilter

Instances: 110 Attributes: 13

State_UT year Primary_Only

Primary_with_U_Primary_Sec_HrSec

U_Primary_With_Sec_HrSec

U_Primary_With_Sec

Sec_with_HrSec.

\author{
HrSec_Only \\ Primary_with_U_Primary \\ U_Primary_Only \\ Primary_with_U_Primary_Sec \\ Sec_Only
}

All Schools

Test mode: 10-fold cross-validation

$===$ Classifier model (full training set) $===$

Bagging with 10 iterations and base learner

weka.classifiers.trees.REPTree -M 2 -V 0.001 -N 3 -S 1 -L -1 -I 0.0

Time taken to build model: 0.03 seconds

$===$ Cross-validation $=====$ Summary $===$

Correlation coefficient $\quad 0.9666$

Mean absolute error

Root mean squared error

Relative absolute error

4.6265

7.7578

$20.3769 \%$

$28.0525 \%$

110 
Vol. 10, Issue 11, November 2021

DOI: $10.17148 /$ IJARCCE.2021.101103

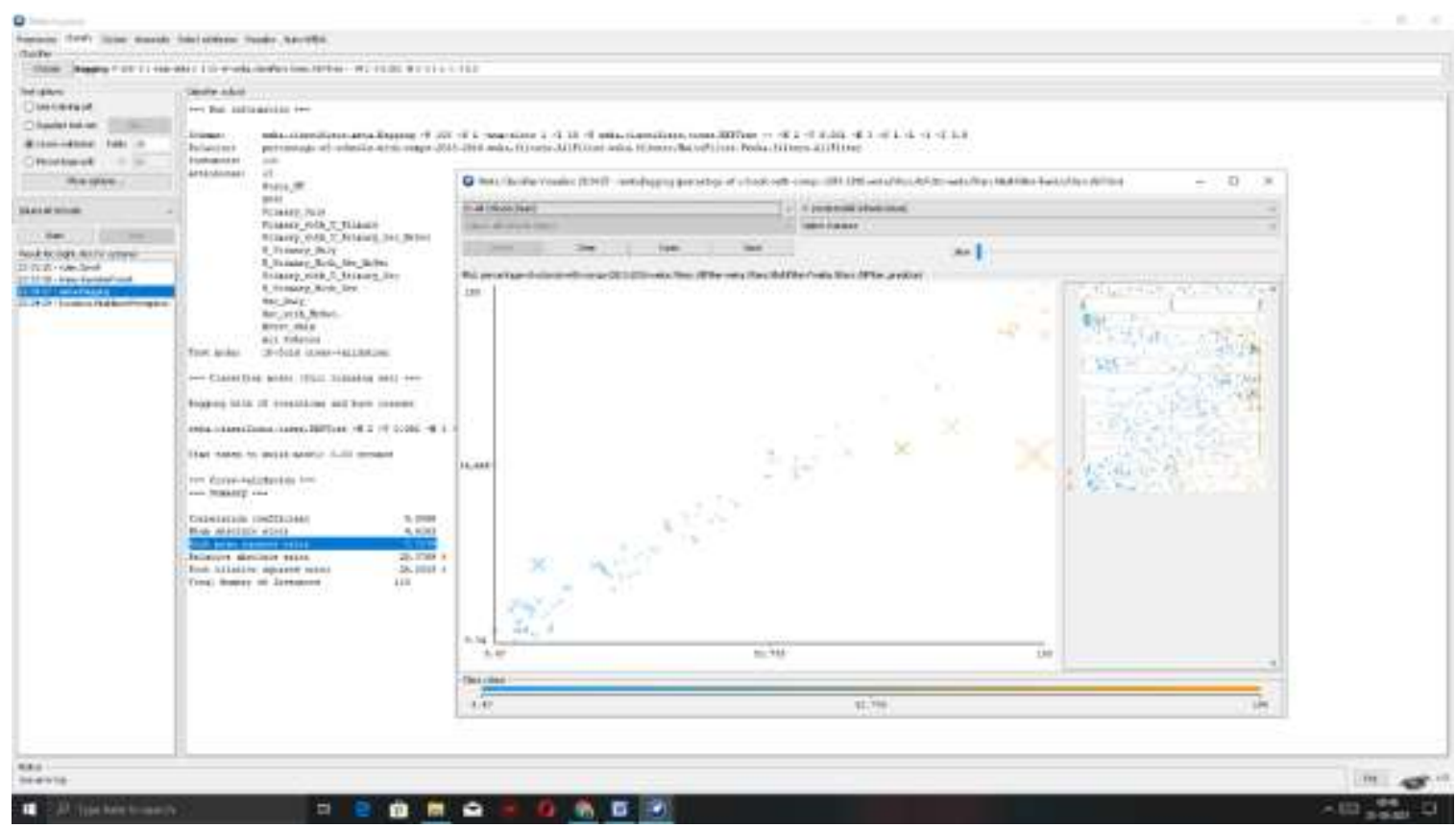

Fig.3 Bagging Classifier Based Prediction with Output Visualization

\subsection{Classifier NAME=weka.classifiers.trees.RandomForest}

SYNOPSIS=Class for constructing a forest of random trees.

Classifier Output=== Run information ===

Scheme: $\quad$ weka.classifiers.trees.RandomForest -P 100 -I 100 -num-slots 1 -K 0 -M 1.0 -V 0.001 -S 1

Relation: percentage-of-schools-with-comps-2013-2016-weka.filters.AllFilter-weka.filters.MultiFilterFweka.filters.AllFilter

Instances: 110 Attributes: 13

State_UT year Primary_Only

Primary_with_U_Primary_Sec_HrSec

U_Primary_With_Sec_HrSec

U_Primary_With_Sec

Sec_with_HrSec.

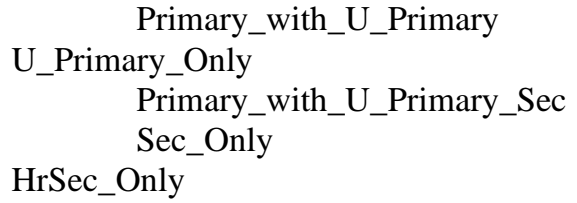

HrSec_Only

All Schools

Test mode: 10-fold cross-validation

$===$ Classifier model (full training set) $===$

RandomForest

Bagging with 100 iterations and base learner

weka.classifiers.trees.RandomTree -K 0 -M 1.0 -V 0.001 -S 1 -do-not-check-capabilities

Time taken to build model: 0.06 seconds

$===$ Cross-validation $=====$ Summary $===$

Correlation coefficient $\quad 0.9931$

Mean absolute error $\quad 3.3593$

Root mean squared error $\quad 4.457$

Relative absolute error $\quad 14.7953 \%$

Root relative squared error $\quad 16.1167 \%$

Total Number of Instances $\quad 110$ 


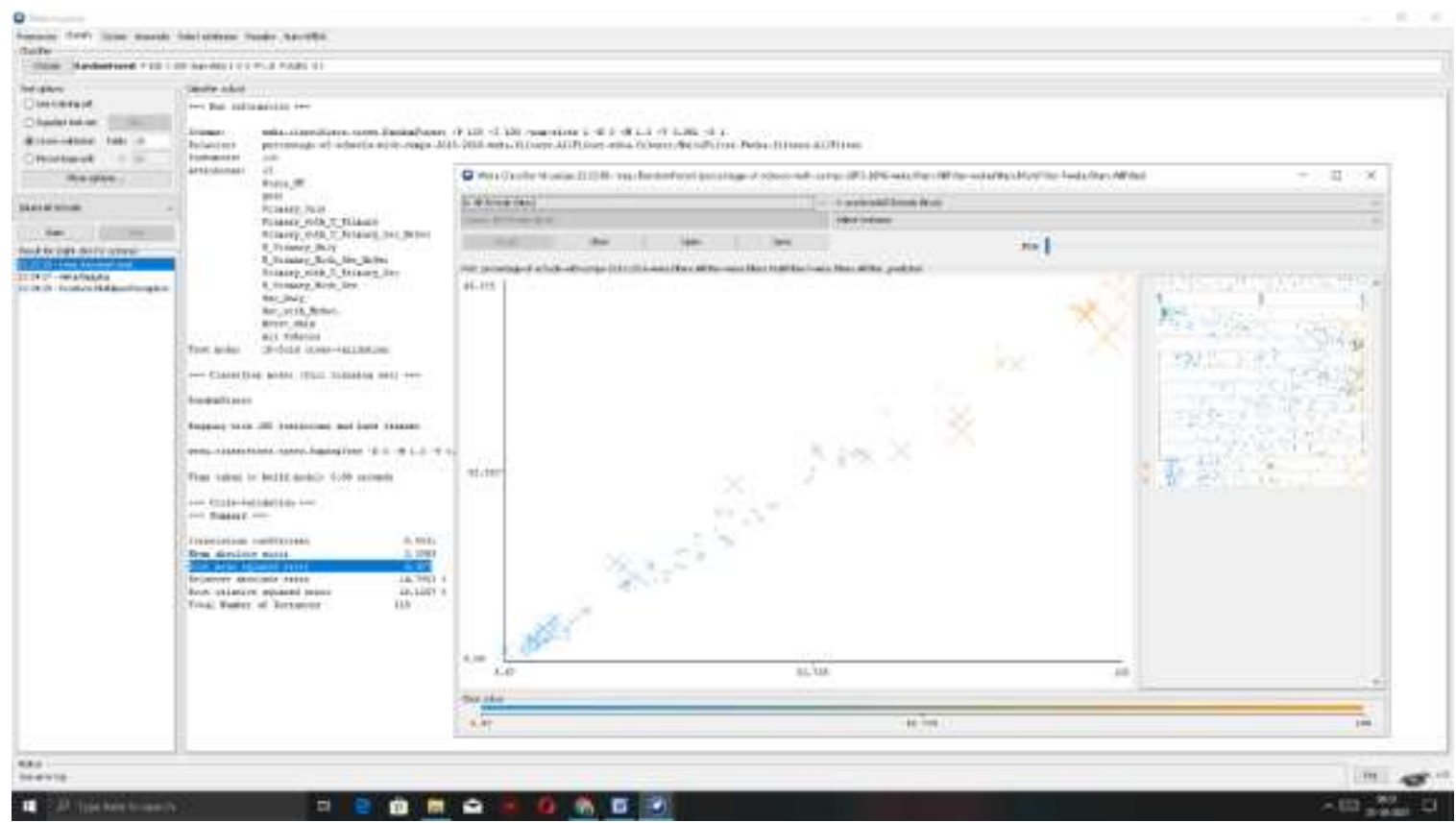

Fig.4 RandomForest Classifier Based Prediction with Output Visualization

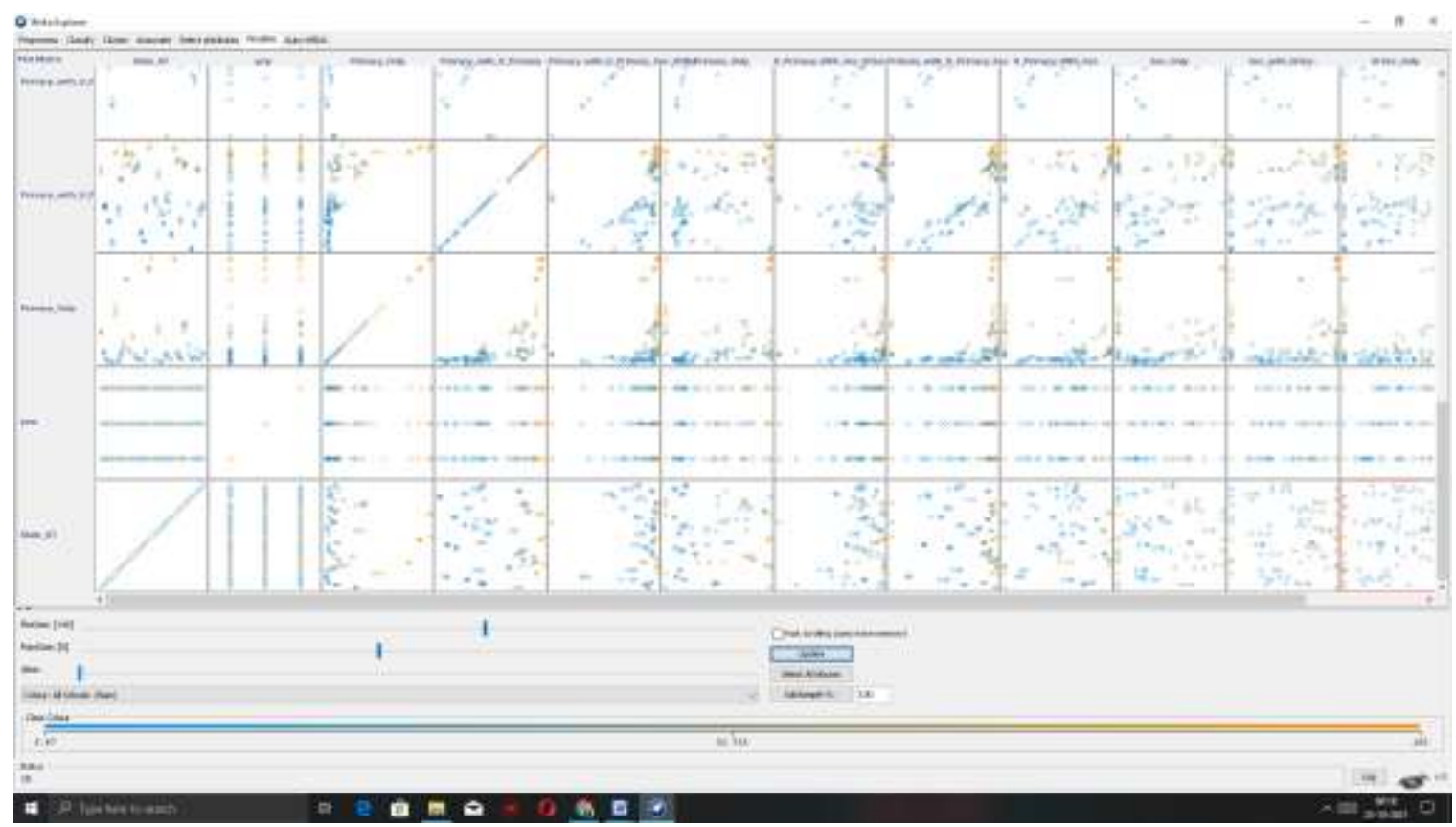

Fig.5 Visualization of \% of Schools with Computers for all Students - Primary Schools to H. Secondary Schools

\section{DISCUSSION}

As we have taken 3 different algorithms and an educational dataset to know the digital and automated cases in different schools in the country. The first classifier that we have taken is the RandomForest in which we got accuracy rate of classified data as $95.543 \%$. The second classifier that we have taken is the metaBagging in which we got accuracy rate of classified data as $92.2422 \%$. The third classifier that we have taken is the MultilayerPerceptron in which we got accuracy rate of classified data as $97.0326 \%$.

Now from this analysis, it is found that number of computers with proper technological infrastructure should be developed gradually to make the educational system towards fully automated digitized and also there are lots of pros and cons regarding whether to make the education system centralized or decentralized. Now it's a great challenge for the higher educational department to make such policies to implement and provide such things to the universities and autonomous educational bodies for the development of the global education system[10]. 


\section{International Journal of Advanced Research in Computer and Communication Engineering}

Vol. 10, Issue 11, November 2021

DOI: $10.17148 /$ IJARCCE.2021.101103

As results from experiments and observation shows that, the Multilayer Perceptron classifier algorithm is an efficient algorithm which gives more accurate results of the correctly classified instances of $97.0326 \%$.

\section{CONCLUSION}

No doubt, educational reforms should be needed time to time as per the demand for the global technological advancements. Education should meet the updated needs of a dynamical inconsistent world, society and new generation students. Decentralized educational reforms should be adopt for a global economical and developed country. Policies are an important concern or a challenge for the higher educational departments to implement decentralized and somewhere centralized time to time based on different criteria. Machine Learning will be an excellent tool to analysis, predict, classify and observation for this type of real issues in the educational reforms and for the digitization of the educational system.

\section{REFERENCES}

[1] Popa, S. Reflections on COVID-19 and the future of education and learning. Prospects 49, 1-6 (2020). https://doi.org/10.1007/s11125-020$\underline{09511-\mathrm{Z}}$

[2] Dalton, L., Rapa, E., and Stein, A. (2020). Protecting the psychological health of children through effective communication about COVID19. Lancet 4, 346-347. doi: 10.1016/S2352-4642(20)30097-3

[3] Ui DS, I Azhar E, Madani TA, Ntoumi F, Kock R, Dar O, et al. The continuing 2019-nCoV epidemic threat of novel coronaviruses to global health - The latest 2019 novel coronavirus outbreak in Wuhan, China. International Journal of Infectious Diseases. 2020;91:264-6. pmid:31953166

[4] Liu, J. J., Bao, Y., Huang, X., Shi, J., and Lu, L. (2020). Mental health considerations for children quarantined because of COVID19. Lancet 4, 347-349. doi: 10.1016/S2352-4642(20)30096-1

[5] Onyema EM, Eucheria NC, Obafemi FA, Sen S, Atonye FG, Sharma A, et al. Impact of Coronavirus pandemic on education. Journal of Education and Practice. 2020;11(13):108-21.

[6] Adnan M, Anwar K. Online Learning amid the COVID-19 Pandemic: Students' Perspectives. Online Submission. 2020;2(1):45-51.

[7] Cao W, Fang Z, Hou G, Han M, Xu X, Dong J, et al. The psychological impact of the COVID-19 epidemic on college students in China. Psychiatry research. 2020;287:112934. pmid:32229390; 32229390.

[8] Sahu P. Closure of Universities Due to Coronavirus Disease 2019 (COVID-19): Impact on Education and Mental Health of Students and Academic Staff. 2020;12(4). pmid:32377489

[9] Poh-Sun G, John S. A vision of the use of technology in medical education after the COVID-19 pandemic. MedEdPublish. 2020;9(1):49. edsdoj.9b8f5410675446488c9f35323d72c0c9.

[10] Latané B. The psychology of social impact. American Psychologist. 1981;36(4):343-56.

[11] Xia S, Liu J. A computational approach to characterizing the impact of social influence on individuals' vaccination decision making. PloS one. 2013;8(4):e60373. pmid:23585835.

[12] Cohen J. Statistical power analysis for the behavioral sciences. 2nd ed: Hillsdale, N.J.: L. Erlbaum Associates; 1988.

[13] Andel SA, de Vreede T, Spector PE, Padmanabhan B, Singh VK, de Vreede G-J. Do social features help in video-centric online learning platforms? A social presence perspective. Computers in Human Behavior. 2020;113. S0747563220302570.

[14] Vargas, C. (2019), "Leaving no one behind: bringing equity and inclusion back into education", Resisting Neoliberalism in Education: Local, National and Transnational Perspectives, L. Tett and M. Hamilton (eds.), Bristol, Policy Press.

[15] Cortis, D. (2020). On determining the age distribution of COVID-19 Pandemic. Front. Public Health 8:202. doi: 10.3389/fpubh.2020.00202

\section{BIOGRAPHY}

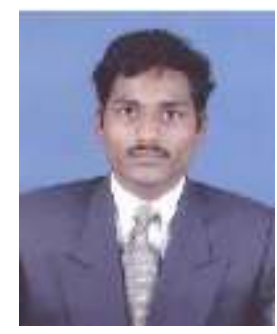

Mr. Dillip Narayan Sahu received his B.Sc. degree in physics from Sambalpur University, India, Master of Computer Application degree from Sambalpur University, India, M.Tech. degree in Computer Science from Sambalpur University, India, M-Phil degree Computer Science from MATS University, India, and continuing Ph.D. in Computer Science. His research area includes Artificial Intelligence, Machine Learning, Analysis and Design of Algorithms, Data Science, IoT. He has published many papers in National/International Conferences, Seminars, and Journals. Presently He is working as a Lecturer in the Dept. of MCA, Gangadhar Meher University, India.

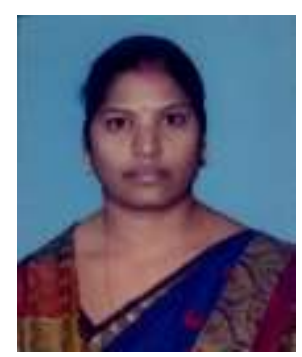

Ms. Pankajini Sahu received her M.Phil. degree in Education from MATS University, India. She has wide publications in many national, International journals and Conferences. Her research area includes Educational Psychology, Primary Education, Learner and learning, Teacher and classroom, Information

and Communication Technology (ICT). Presently she is working as a Lecturer in Dept. of Education, ShreeRam College, Rampur, Sonepur, Odisha, India. 